\title{
Therapeutic Targets of KRAS in Colorectal Cancer
}

\author{
Shafia Rahman ${ }^{1}$, Shimon Garrel ${ }^{2}$, Michael Gerber ${ }^{3}$, Radhashree Maitra ${ }^{1,3}$ (i) and Sanjay Goel ${ }^{1, *(1)}$ \\ 1 Department of Medical Oncology, Montefiore Medical Center/Albert Einstein College of Medicine, \\ 1695 Eastchester Road Bronx, New York, NY 10461, USA; shafrahman@montefiore.org (S.R.); \\ RMAITRA@montefiore.org (R.M.) \\ 2 Department of Biology, Lander College For Men, 75-31 150th Street, Flushing, New York, NY 11367, USA; \\ sgarrel@student.touro.edu \\ 3 Department of Biology, Yeshiva University, 500 West 185th Street, New York, NY 10033, USA; \\ mgerber@mail.yu.edu \\ * Correspondence: SGOEL@montefiore.org; Tel.: +1-7184304136
}

Citation: Rahman, S.; Garrel, S.; Gerber, M.; Maitra, R.; Goel, S. Therapeutic Targets of KRAS in Colorectal Cancer. Cancers 2021, 13, 6233. https://doi.org/10.3390/ cancers 13246233

Academic Editor: Chiara Ambrogio

Received: 28 October 2021

Accepted: 9 December 2021

Published: 11 December 2021

Publisher's Note: MDPI stays neutral with regard to jurisdictional claims in published maps and institutional affiliations.

Copyright: (c) 2021 by the authors. Licensee MDPI, Basel, Switzerland. This article is an open access article distributed under the terms and conditions of the Creative Commons Attribution (CC BY) license (https:/ / creativecommons.org/licenses/by/ $4.0 /)$.
Simple Summary: Colorectal cancer is among the most common cancers in the United States. The advancement in treatment and early diagnosis have enabled a reduction in mortality from the disease among the patients with early and localized disease; however, the survival continues to be dismal in the metastatic colorectal cancers. Understanding the biological and genetic factors is crucial is making the therapeutic strategy and improving survival outcomes. One of such critical steps is the understanding of the mechanism and development of therapeutic targets against metastatic colorectal cancers bearing the KRAS mutation.

Abstract: Patients with metastatic colorectal cancer have a 5-year overall survival of less than $10 \%$. Approximately $45 \%$ of patients with metastatic colorectal cancer harbor KRAS mutations. These mutations not only carry a predictive role for the absence of response to anti-EGFR therapy, but also have a negative prognostic impact on the overall survival. There is a growing unmet need for a personalized therapy approach for patients with KRAS-mutant colorectal cancer. In this article, we focus on the therapeutic strategies targeting KRAS- mutant CRC, while reviewing and elaborating on the discovery and physiology of KRAS.

Keywords: colorectal cancer; KRAS mutation; targeted therapy

\section{Introduction}

Colorectal cancer (CRC) is one of the most common cancers, with an estimated 1.5 million new cases and 52,980 deaths reported in 2021 in the United States, of which approximately 104,270 arise from the colon and the remainder from the rectum [1]. Although the mortality related to the CRC has been progressively declining since 1990, it continues to be the third most common cause of cancer death in both men and women, respectively, in the United States [2].

CRC arises through a multistep process involving accumulation of various epigenetic and genetic alterations [3]. The pathogenic mechanisms implicated in $80-85 \%$ of all CRC cases include microsatellite instability (MSI), CpG island methylator phenotype (CIMP), and chromosomal instability (CIN). The CIN is the most common pathogenic mechanism involved in the development of CRC. It results in the gain or loss of entire or large portions of chromosome resulting in karyotype changes within the cells. These karyotypic changes coupled with the mutations in the tumor suppressor and oncogenes (APC, KRAS, DCC/SMAD4, and TP53) activate oncogenic pathways critical to the pathogenesis of CRC [4]. Mutations in any of the four mismatch repair genes ( $h M L H 1, h M S H 2, h M S H 6$, and $h P M S 2)$ result in the microsatellite instability and leads to the development of the hereditary nonpolyposis colorectal cancer (HNPCC), also known as Lynch syndrome [5]. This genetic disorder is inherited as an autosomal dominant pattern and increases the 
risk of development of several cancers involving the colon, stomach, prostate, and small intestine [4]. The $\mathrm{CpG}$ island methylator phenotype is a unique subgroup of CRC. It is characterized by the epigenetic instability resulting in the hypermethylation of $\mathrm{CpG}$ island sites at the promoter regions that sequentially leads to transcriptional inactivation of tumor suppressor genes in CRC [5]. In the majority of the cases, one pathway is the predominant pathogenic pathway; however complex interplay in certain cases could be seen [6]. Apart from the above described pathogenic mechanisms, recent studies have shown the alteration in the various metabolic pathways including glycolysis, pyruvate oxidation, lactate oxidation, mitochondrial activity, and glutamine and cholesterol metabolism are involved in the initiation and progression of CRC [7].

The RAS gene family is mutated in approximately $30 \%$ of human cancers, with the KRAS isoform mutations being the major contributor [8-10]. In Colon cancers, approximately $45 \%$ of the cases carry a KRAS mutation [11,12]. These mutations in CRC are associated with aggressive tumor biology and poor survival. Moreover, the KRAS mutations in CRC lead to resistance to epidermal growth factor receptor (EGFR) directed therapies [13].

\section{Discovery of RAS}

Over six decades ago, Jennifer Harvey and Werner Kristen identified the two RAS gene, $H R A S$ and KRAS, from the Harvey sarcoma virus and Kirsten sarcoma virus, respectively. The viral inoculum from leukemic rats was observed to induce sarcoma in newborn rats [14,15]. Later, Stehelin et al. proved that these proto-oncogenes could transform into oncogenes after acquiring mutations and these oncogenes can be virally transmitted [16]. Subsequently, in 1982, the human HRAS and KRAS oncogene sequences were ascertained in human bladder and lung cancer cell lines, respectively [17]. Later in 1983, the human sequences homologous to NRAS were described in human sarcoma cell lines [18]. Overall, the RAS family constitutes 36 human genes, but KRAS, HRAS, and NRAS by far are the most prominent ones involved in human cancer [19]. These high occurrences make $R A S$ one of the most critical targets in oncology for drug development.

\section{Physiology of RAS}

KRAS is located at 12p12.1 and encodes a 188-amino acid residues [20]. The RAS family of genes acts as a universal confluence in the signal transduction of multiple intracellular pathways. KRAS and other RAS oncogenes are intracellular guanine nucleotide-binding proteins (G proteins) that belong to the family of small GTPases and function as GDP/GTPregulated molecular switches [21]. It is activated by varying signals ranging from growth factors (epidermal growth factor receptor, platelet-derived growth factor receptor, insulin like growth factor, etc.), hormones, and cytokines to neurotransmitters [20]. Once activated, RAS moves from an inactivated, GDP-bound form, to activated GTP- bound state. The activation is catalyzed by guanine nucleotide exchange-factors (GEFs) and the conversion back to inactivated form by GTP hydrolysis mediated by GTPase-activating proteins (GAPs) [22]. The RAS activates multiple downstream pathways including the RAS-RAFMEK-ERK pathway, which regulates cell cycle and proliferation [22]. Another pathway involved is PI3K-AKT-mTOR, which also promotes cell growth and suppresses apoptosis. The RAS-related protein (RAL) pathway and the tumor invasion and metastasis-inducing protein 1 (TIAM1-RAC1) are involved in intracellular vesicle trafficking, cytoskeletal organization, and tumor growth [23]. Thus, RAS proteins are essential regulators of the various aspects of normal cell growth and physiology and play a role in malignant transformation (Figure 1). Apart from playing critical role in the signal transduction involving multiple intracellular pathways, oncogenic KRAS is known to dysregulate various metabolic processes including glutaminolysis, glycolysis, and redox hemostasis promoting tumorigenesis and chemoresistance [24]. 


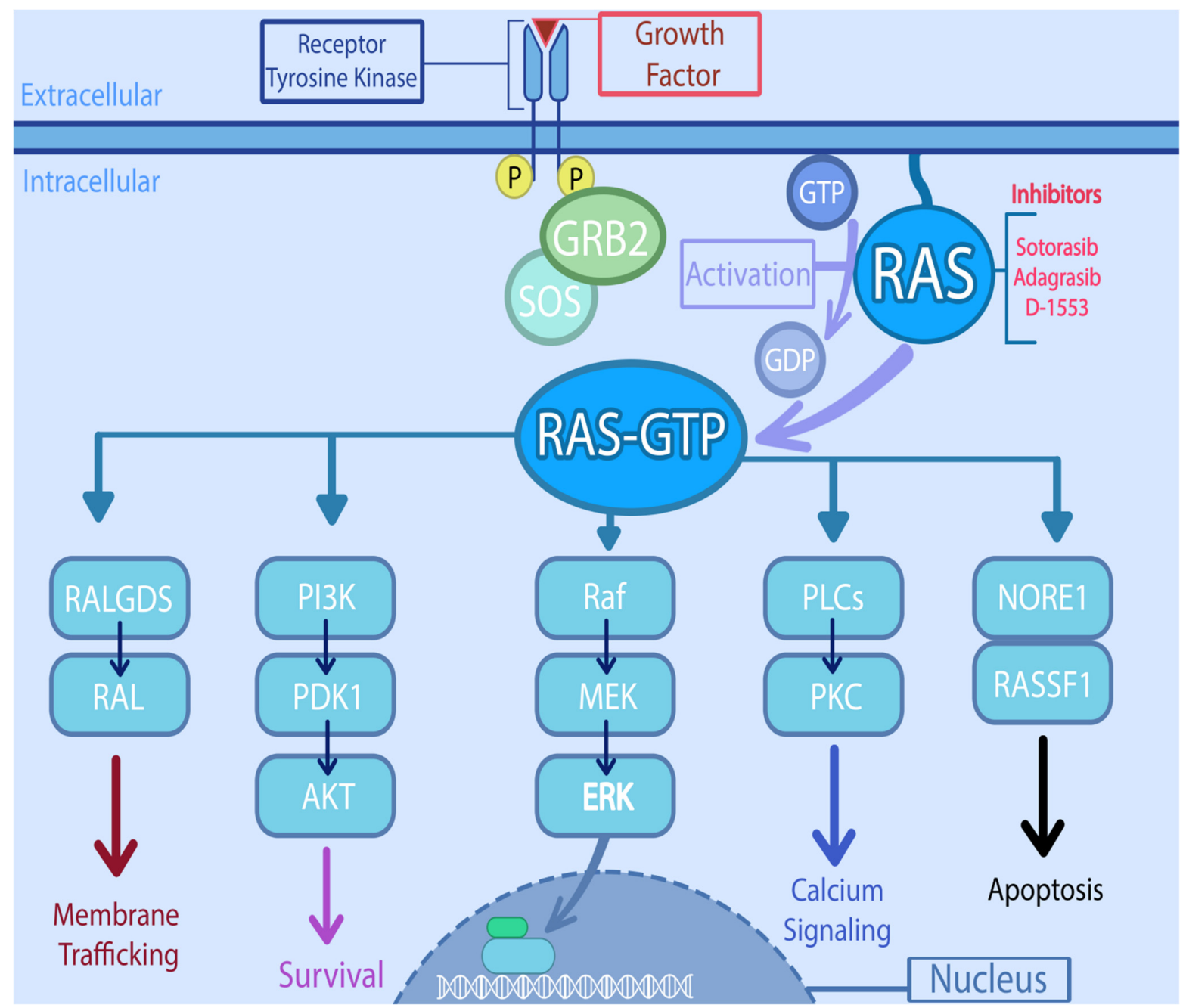

Figure 1. Signaling pathways downstream of RAS and potential targets. RAS directly activates the mitogen activated protein kinase (MAPK) cascade, through phosphorylation of Raf (Rapidly accelerated fibro sarcoma) which in turn phosphorylates MEK (Mitogen activated protein kinase kinase), which then phosphorylates MAPK. On the other hand, it also interacts with the PI3K (Phosphatidylinositol-4,5-Bisphosphate 3-Kinase)/AKT (serine/threonine protein kinase) pathway, either by PI3K interaction or through RAC1 which in turn activates p21-activated kinase (PAK), an AKT interacting protein. RAS also activates the RAL (RAS like proto-oncogene) which is involved in various steps of membrane trafficking. The PLCs (Phospholipase C) along with RINI/ABL plays important role in cytoskeletal remodeling. The activation of NORE1/RASSF1 is involved in cell cycle arrest and apoptosis.

\section{Mutations Involving RAS in CRC}

RAS mutations have been associated with aberrant cell signaling that leads to tumorpromoting inflammation and play a key role in carcinogenesis by inducing an array of inflammatory cytokines, chemokines and accentuates tumorigenesis and invasiveness. The RAS mutations are common in CRC $(\sim 45 \%)$, with KRAS being the most prevalent $(85 \%)$, followed by NRAS (15\%) and HRAS (1\%) [25]. The majority of the KRAS mutations in the CRC are located in codons 12 and 13 of exon $2(80 \%$ are G12A, G12C, G12D, G12S, G12V, G13C, G13D), and less frequently in codon 61 of exon $3(5 \%$ are Q61H, Q61L, and Q61R) and codon 146 of exon 4 ( $2 \%$ are A146T and A146V) [26]. Mutations in any of these codons promote the accelerated exchange of nucleotides, and a decrease in the binding of GAP. Either of these leads to increase GTP binding and KRAS activation. KRAS mutations also carry a predictive role for the absence of response to anti-EGFR therapy in metastatic CRC and thus have a negative prognostic impact as well $[27,28]$. 


\section{Targeting KRAS}

The therapeutic strategies under investigation to target KRAS mutations in CRC includes therapy directed towards mutant $K R A S$, targeting KRAS-membrane association, and the combined inhibition of downstream pathways.

\subsection{KRAS Directed}

Several studies are being performed to identify molecules able to bind the mutated sites of KRAS or inhibit the synthesis at the DNA level of the mutated protein and subsequently blocking the activity of KRAS.

\section{2. $A M G 510$}

AMG 510 (Sotorasib) is the first FDA-approved specific, irreversible inhibitor of KRAS G12C. It traps the KRAS in the inactive GDP-bound state [29]. AMG 510 has shown in the preclinical studies to inhibit phosphorylation of extracellular signal-regulated kinase (ERK), a critical downstream effector of $K R A S$, producing a durable complete tumor regression in mice bearing KRAS p.G12C tumors [30]. Based on the significant objective response rate and duration of response in a phase 1 trial CodeBreak100 (NCT03600883) [31], it was approved in locally advanced or metastatic NSCLC. Although the KRAS G12C is noted only in $1-3 \%$ of $C R C$, the recent promising clinical data breaks the assumption of KRAS being undruggable [32].

\subsection{MRTX849}

Another direct target of KRAS is MRTX849 (adagrasib). It works by irreversibly and selectively binding to KRAS G12C in its inactive state, blocking its signaling to other cells, thus preventing tumor cell growth and proliferation, leading to cancer cell death [33]. KRYSTAL-1 phase I/II clinical trial showed clinical activity in non-small cell lung cancer (NSCLC), CRC and other solid tumors such as pancreatic, endometrial, and ovarian cancers [34]. The FDA granted breakthrough therapy designation to MRTX849 for the treatment of patients with KRAS G12C-mutated non-small cell lung cancer patients that has previously received systemic therapy.

\subsection{MRTX1133}

MRTX1133. is another KRAS directed investigational drug. The preclinical studies have demonstrated that it selectively inhibits the KRAS G12D mutant forms, binds both the active and inactive forms and significant dose dependent tumor regression was noted in the animal models. The phase $1 / 1 \mathrm{~b}$ study and phase 2 monotherapy trial in patients with NSCLC showed ORR of $45 \%$, with mean duration of treatment being greater than 6 months [35,36].

Few other direct KRAS inhibitors targeting KRAS G12C mutation in phase 1 clinical trials include GDC-6036 (NCT04449874), JNJ-74699157 (NCT04006301), and D-1553 (NCT04585035) [37].

\subsection{PLK-1 Inhibition}

Polo-like kinase 1 (PLK1) is a serine/threonine kinase which plays a key role both in cell cycle progression via mitosis and DNA damage repair [38]. It has been found to be overexpressed in multiple cancer types including CRC [39]. Several studies have suggested correlation between Plk1 overexpression and poor prognosis. This has resulted in the development and emergence of PLK-1inhibtors as next generation anti-cancer therapy [40]. It has been observed that the RAS mutant cells are dependent on gene/proteins such as PLK-1, which are involved cell proliferation [41]. One such drug is Onvansertib, which is a selective competitive inhibitor PLK-1 inhibitor [42]. It is under clinical investigation as a second line therapy in metastatic CRC harboring KRAS mutation along with combination of FOLFIRI and bevacizumab (Clinicaltrials.gov Identifier: NCT03829410). Preliminary data from this Phase1b/2 study, which was presented at the American Society of Clinical Oncol- 
ogy (ASCO) Gastrointestinal symposium, showed that $42 \%$ of patients achieved a partial response (PR) and a durable response in 67\% ranging from 6.1 months to 13.7 months [43].

\subsection{KR12}

Another promising drug for colorectal patients with either the G12D or G12V mutation is KR12. This agent is a pyrrole-imidazole polyamide indole-seco-CBI conjugate) which recognizes and alkylates the adenine residues on the template strand at codon 12 (GTT and GAT), exon 2 of mutated KRAS, producing strand cleavage which in turn decreases the proliferation rate of the CRC cell harboring G12D/G12 V mutation [44]. The growth suppression in G12D/G12V mutated CRC cells, ultimately induced senescence, and apoptosis. This effect was demonstrated in the preclinical study performed by Nagese et al. [44]. KR12 also induced significant tumor growth suppression in xenograft models, with low host toxicity in KRAS-mutated but not wild-type tumors, thus representing a promising agent against RAS mutated CRC with encouraging pre-clinical data.

\subsection{SHP2 Inhibition}

Src homolofy-2 Domain containing protein tyrosine phosphatase-2 (SHP2) is encoded by human PTPN11 gene and acts as a protein tyrosine phosphatase [45]. It is involved in various intracellular oncogenic signaling pathways, including the RAS/Raf/MAPK [46,47], PI3K/AKT [48], Jak/STAT [49], PD-1/PD-L1 [50], and mTOR pathways [51]. It functions as a protein tyrosine phosphatase that removes the tyrosine phosphorylation which is a crucial catalytic action and plays an important role in the multiple cellular functions including cellular proliferation, differentiation, and migration [52]. Overall, the primary oncogenic role of the SHP2 in the activation of the RAS/Raf pathway is to cause dephosphorylation of the tyrosine residues in the scaffolding proteins that result in the increased conversion of inactive RAS (RAS-GDP) to the activated RAS (RAS-GTP) [53]. This catalytic function also makes SHP2 a critical facilitator in acquired resistance of the RAS signaling reactivation, to overcome pharmacological inhibition [54]. These properties promote SHP2 inhibition as an attractive way to combat adaptive resistance, both as a monotherapy as well as and in combination with other agents such as, MEK inhibitors. Currently, four SHP2 inhibitors under investigation and are undergoing phase I clinical trials: JAB-0368 (NCT03518554), TN0155 (NCT03114319), RMC-4630 (NCT03634982), and RLY-1971 (NCT04252339). Table 1 depicts the KRAS directed drugs under clinical investigation involving metastatic CRC.

Table 1. Ongoing KRAS directed clinical trials involving metastatic CRC.

\begin{tabular}{|c|c|c|c|c|c|}
\hline Clinical Trial & Drug & Target & Cancer Type & $\begin{array}{c}\text { Estimated } \\
\text { Enrollment (N) }\end{array}$ & NCT ID \\
\hline Phase I & MRTX849 & KRAS G12C inhibitor & $\begin{array}{l}\text { KRAS G12C mutant } \\
\text { cancers }\end{array}$ & 565 & NCT03785249 \\
\hline Phase 1 & KRAS TCR & $\begin{array}{l}\text { Anti-KRAS G12D } \\
\text { engineered T-cells }\end{array}$ & $\begin{array}{c}\text { KRAS G12D } \\
\text { Mutated cancer }\end{array}$ & 70 & NCT03745326 \\
\hline Phase 1 & KRAS TCR & $\begin{array}{l}\text { Anti-KRAS G12 V } \\
\text { engineered T-cells }\end{array}$ & $\begin{array}{c}\text { KRAS G12V } \\
\text { Mutated cancer }\end{array}$ & 110 & NCT03190941 \\
\hline Phase 1 & $\begin{array}{c}\text { GDC-6036+/- } \\
\text { Atezolizumab, } \\
\text { Cetuximab, } \\
\text { Bevacizumab, Erlotinib }\end{array}$ & KRAS G12C Mutation & $\begin{array}{c}\text { Advanced or } \\
\text { Metastatic Solid } \\
\text { Tumors With a KRAS } \\
\text { G12C Mutation }\end{array}$ & 342 & NCT0444987 \\
\hline Phase 1 & BВP-398 & SHP2 inhibitor & $\begin{array}{c}\text { MAPK pathway or } \\
\text { RTK driven advanced } \\
\text { solid tumors }\end{array}$ & 60 & NCT04528836 \\
\hline Phase $1 b / 2$ & $\begin{array}{c}\text { Onvansertib (PCM-075) } \\
+ \text { FOLFIRI + } \\
\text { bevacizumab }\end{array}$ & PLK-1 inhibitor & $\begin{array}{l}\text { Metastatic CRC with } \\
\text { KRAS mutation }\end{array}$ & 44 & NCT03829410 \\
\hline
\end{tabular}


Table 1. Cont.

\begin{tabular}{|c|c|c|c|c|c|}
\hline Clinical Trial & Drug & Target & Cancer Type & $\begin{array}{c}\text { Estimated } \\
\text { Enrollment (N) }\end{array}$ & NCT ID \\
\hline Phase $1 b / 2$ & SX-682 +/-nivolumab & CXCR1/2 inhibitor & $\begin{array}{c}\text { Metastatic CRC, RAS } \\
\text { mutated }\end{array}$ & 53 & NCT04599140 \\
\hline Phase 1 & JNJ-74699157 & KRAS G12 C & $\begin{array}{c}\text { KRAS mutated } \\
\text { advanced solid tumor }\end{array}$ & 10 & NCT0400630 \\
\hline Phase 1 & $\begin{array}{c}\text { mRNA-5671/V941 } \\
+/ \text {-pembrolizumab }\end{array}$ & $K R A S$ vaccine & $\begin{array}{l}\text { KRAS mutant CRC, } \\
\text { NSCLC and PDAC }\end{array}$ & 100 & NCT03948763 \\
\hline Phase 1 & D-1553 & KRAS G12C inhibitor & $\begin{array}{l}\text { KRAS mutated CRC } \\
\text { and NSCLC }\end{array}$ & 200 & NCT04585035 \\
\hline
\end{tabular}

\section{Targeting Membrane Association}

Targeting of G4 Structures

G-quadruplex (G4) structures are DNA tetraplexes that typically form in guanine-rich regions of genomes. Four guanine bases bind with Hoogsteen hydrogen bonds to form a guanine tetrad plane (G-quartet), and then two or more G-quartet planes stack on top of each other to form a G4 structure [55] Figure 2. The G4 structures are abundant in the promoter regions of many genes, including the regulation transcription of oncogenes and tumor suppressor genes [56-58]. Other than being reported on the KRAS human promoter DNA, G4 structures are found in RNA sequences, including the $5^{\prime}$ untranslated region (UTRs) of KRAS mRNA. Purro et al. identified natural alkaloids Indoloquinolines as potential G4-ligand compounds for targeting of KRAS in CRC [59,60]. They also synthesized a new molecule, namely EMICORON, which binds to the G4 structures on KRAS. Treatment with EMICORON, downregulated KRAS mRNA and protein expression in CRC cell lines, with decreased tumor volume in KRAS-mutated patient-derived xenografts [59]. It was further shown the EMICORON co- administration with FOLFIRI, improved the efficacy of chemotherapy in CRC-bearing mice [59].

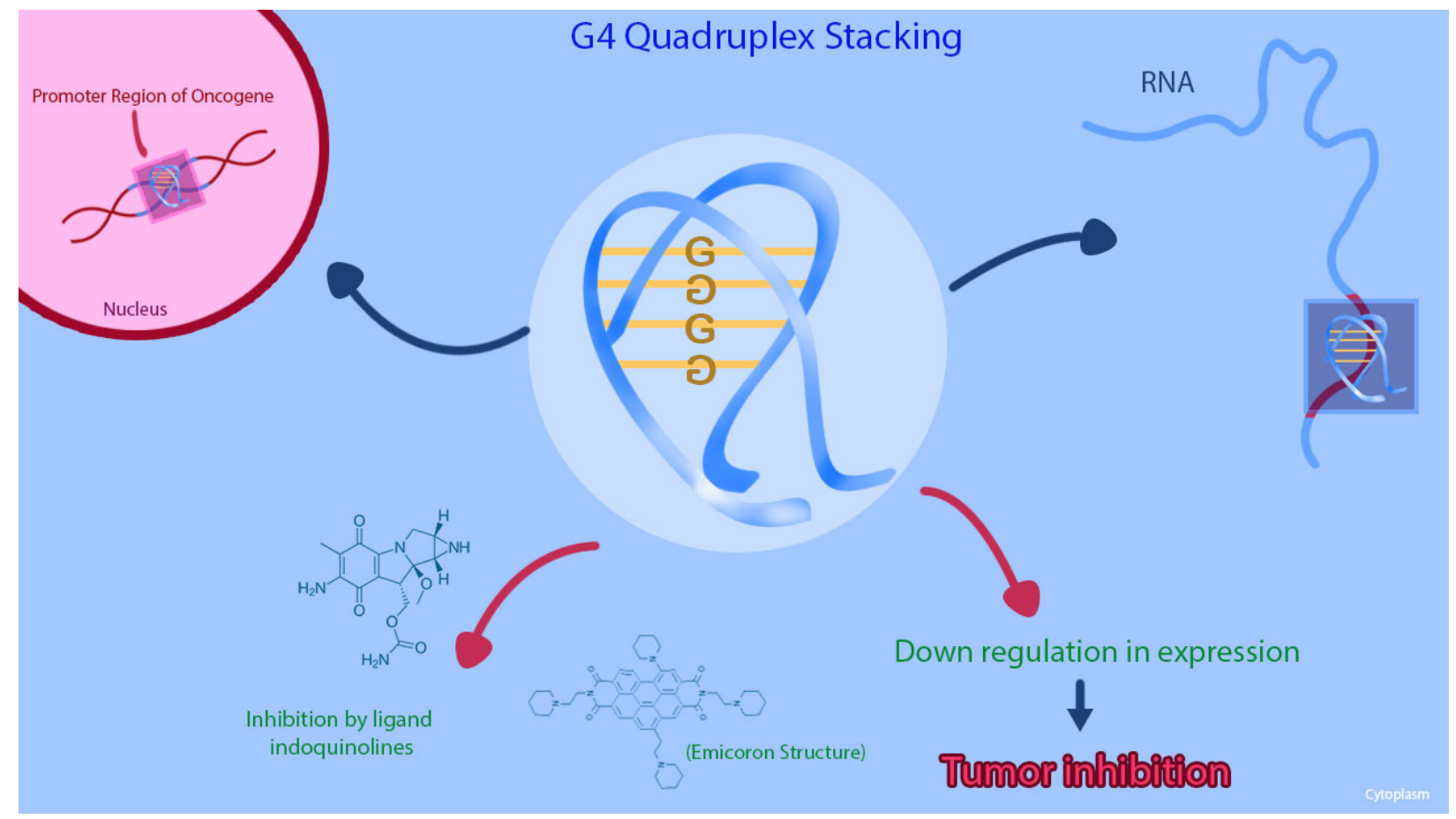

Figure 2. This figure shows that the G-quadruplex (G4) structures and the inhibition of the G4 structure on KRAS promoter region can cause downregulation of gene expression in CRC cell lines. 


\section{Indirect Approaches}

\subsection{PDE Inhibition}

Prenyl-binding protein PDE $\delta$ is crucial in maintaining the spatial organization of RAS during the activation of signaling pathway [61,62]. This creates a novel mechanism of indirectly targeting RAS signaling through inhibition of PDE $\delta$. Zimmermann et al., developed Deltarasin, a small molecule inhibitor of PDE $\delta$ and demonstrated in the preclinical study that PDE $\delta$ inhibition by Deltarasin not only blocked the oncogenic RAS signaling but suppressed both in vitro and in vivo proliferation of human pancreatic ductal adenocarcinoma cells with highly prevalent oncogenic KRAS mutant genes [58]. Based on this pre-clinical data various small molecule inhibitors of PDE $\delta$ were generated. These inhibitors, such as Deltazinone 1 and Deltasonamide 1 and 2, competitively interact with the farnesyl-binding pocket of RAS [63,64]. Another pre-clinical study supporting the above strategy was done by Klein et al., [65]. They successfully demonstrated the inhibitory effect of PDE $\delta$ blockage on the proliferation and survival of KRAS mutant CRC cell lines.

\subsection{Targeting NRF2/Oxidative and Glutaminolysis}

One of the major cellular changes which drive the proliferation in the cancer cells is the ability to induce metabolic reprogramming [63]. The normal cells induce glycolysis as a source of ATP in response to hypoxia. However, the cancer cells express exorbitant aerobic glycolysis promoting rapid cellular proliferation. This phenomenon is known as the Warburg's effect [64]. One of the major sources of substrates for anerobic glycolysis is glutamine. Glutamine is an important amino acid necessary for the synthesizing glutamate via glutaminolysis, which in turn contributes to the tricarboxylic acid (TCA) cycle in the absence of glucose [65], thus making the cancer cells dependent on the glutamine-mediated TCA cycle for their energy needs. However, the rapid cellular proliferation and metabolism rate generates oxidative radicals deleterious for cell survival. The nuclear factor-erythroid 2 -related factor 2 (NRF2) and Kelch-like ECH-associated protein 1 (KEAP1) pathway protects the cells against oxidative and electrophilic stress and is tightly regulated under normal physiological conditions [66]. In cancer cells, apart from the enhanced glutaminolysis, dysregulation of NRF2/oxidative, inhibition of repressor genes, pathway promotes constant detoxification and transcription of cytoprotective proteins. Multiple experimental studies have shown that mutant KRAS enhances the activation of NRF2 antioxidant system and gene expression of the enzymes involved in the glutaminolysis, promoting tumorigenesis $[67,68]$. Given this critical role of glutaminolysis and NRF2/oxidative pathway, it has become an exciting therapeutic target to combat KRAS driven cancers. Furthermore, the glutaminase and NRF2 inhibitors have shown to enhance sensitivity of cancer cells to chemotherapy. Mukhopadhyay et al. showed that the NRF2 contributed to chemoresistance in KRAS mutated pancreatic cancer cells and targeting these cell lines with glutaminase inhibitors sensitized the KRAS mutant cells to chemotherapy [69]. The glutaminase inhibitors under investigation so far include DON, JHU-083, BPTES, CB-839, and compound 968; however, the exuberant metabolic heterogenicity enhances the complexity of these targeted small molecule inhibitors [70,71]. Direct NRF2 inhibitor is another approach to sensitize KRAS mutant tumors to chemotherapy. One of the agents under investigation is brusatol and has shown promising data in pre-clinical studies [72,73].

\subsection{Oncolytic Virus Induced Autophagy}

Pelareorep is oncolytic reoviruses and has been under investigation as a therapeutic cancer-directed agent for over a decade [66]. Pelareorep can selectively infect the KRAS mutated CRC cells inducing lysis and promoting autophagy [67]. This was demonstrated in the pre-clinical study conducted by Maitra et al. [68]. In a phase I clinical trial, pelareorep was combined with FOLFIRI/Bevacizumab, which showed the combination was able to induce $50 \%$ partial response in the enrolled patient cohort [69]. Given the encouraging results, further exploration of this combination is needed as a modality for KRAS directed therapy. 


\section{Combined Inhibition of Downstream Pathways}

The RAS/RAF/MEK/ERK and PI3K/AKT/mTOR are two of the most frequently dysregulated pathways in the human cancer biology [70]. These two pathways interact closely, not only sharing common inputs but are also activated by oncogenic RAS. These pathways also provide compensatory signaling when either one is inhibited [71]. This provides the scientific basis for combining therapeutic agents that could simultaneously block both the pathways and inhibit the downstream pathways involved in RAS signaling.

\subsection{AZD4785}

AZD4785 is a genetically engineered molecule which functions as an antisense oligonucleotide complementary to mRNA sequences of KRAS and selectively exhausts the intracellular KRAS mRNA and protein. The depletion of KRAS protein results in the repression of the subsequent signaling pathways and thus suppresses the cell proliferation. The KRAS depletion was not associated with any reciprocal feedback activation of MAPK pathway [72]. The other feature of AZD4785 is that its codon binding site on the KRAS mRNA is different from the mutation codon sites, thus it could be an effective strategy to target both wild and mutant type KRAS. Another pre-clinical study using AZD4785 on a panel of various tumor cell lines including CRC showed potent downregulation of mutant KRAS [73]. However, a Phase 1, dose escalation study of AZD4785 in patients with advanced solid tumors did not demonstrate sufficient KRAS lowering in target engagement, prompting the termination of therapeutic development (ClinicalTrials.gov Identifier: NCT03101839).

\subsection{AZD6244}

AZD6244 (Selumetinib) is an oral selective mitogen-activated protein kinase kinase (MAPKK, or MEK) pathway inhibitor and targets MEK1 and MEK2 [74]. The selumetinib interacts with MEK1/2 by turning MEK1/2 into their inactive conformational states. The inactive MEK $1 / 2$ can undergo ATP and substrate binding, but disrupts the interactions required for extracellular signal-related kinase (ERK) activation. Pre-clinical studies with AZD6244 have shown regression of tumors both in KRAS wild-type BxPC3 pancreatic tumor xenograft model, as well as in colorectal, pancreatic, non-small cell lung, hepatocellular, and melanoma human xenograft models [75]. Bennouna et al. performed a Phase II study wherein AZD6244 therapy was compared to capecitabine monotherapy in CRC patients refractory to oxaliplatin and irinotecan. This study showed that selumetinib was well tolerated, and efficacy was comparable to capecitabine [76]. Apart from CRC, selumetinib has been tested in Phase II setting in various gastrointestinal tumor types including HCC, biliary cancer, and pancreatic cancer [77]. Currently, Phase II trials in combination with chemotherapy are undergoing in KRAS mutant CRC tumor types.

\subsection{MEK and P13K/mTOR Combination}

Combined Inhibition of downstream RAS signaling pathways such as MEK, P13K, and mTOR represents an effective strategy in KRAS mutant CRC. A phase 1 clinical study conducted by Shimizu et al. showed combined blockage using a PI3K pathway inhibitor in combination with a MAPK pathway inhibitor in advanced solid tumors including CRC observed tumor regression ranging between $2 \%$ and $64 \%$ [78]. A pre-clinical study supporting the above results was done by Pitt et al. where they demonstrated suppression of tumor progression in CRC cell lines and tumor xenografts models with combinatory PI3K/mTOR inhibitor PF-502 and the MEK1/2 inhibitor PD-901 [79]. Similar to these studies, there are numerous pre-clinical and early phase studies showing the synergistic effect of MEK and P13K/mTOR [80,81].

\section{Developing Therapies}

\subsection{MiRNA as Potential Drug Candidates}

MicroRNAs (miRNA) are small, approximately 20-nucleotide long, single stranded, non-coding RNA molecules and regulate gene expression by binding to complementary 
$3^{\prime}$ untranslated region (UTR) of a target gene leading to either degradation of mRNA or inhibition of translation [82]. Multiple studies have elucidated the critical role of miRNAs in cell proliferation, migration, invasion, apoptosis, and angiogenesis [83]. These miRNAs can function as either a tumor suppressor or an oncogene in the regulation pathway, depending on the cell context. For example, an miR-96 is upregulated in lung, prostate, bladder, colorectal, and breast cancer however the same miR-96 is downregulated in pancreatic cancer [84]. Chan et al. experimentally showed that miR-143 downregulation was associated with upregulation of KRAS protein in CRC cell lines. Upon treating CRC cell lines with miR-143, suppression of KRAS protein translation was observed. However, when miR-143 inhibitor was used it stimulated cell proliferation and increased the KRAS protein level [85]. In another study, Zhou et al. identified FAK and LAMB3 as targets of miRNA-1298 and observed that an miRNA-1298 mimic was noxious to CRC and NSCLC mutant cells in both vitro and in vivo conditions [86]. The regulatory role of miRNA on KRAS in various tumor types including CRC makes miRNAs a fascinating emerging drug therapy.

\subsection{MYC Inhibition}

The RAS and the MYC oncogenes interplay and interdependency play an essential role in driving cancer development [87]. Several studies in mouse models have demonstrated the importance of MYC in KRAS-driven oncogenesis and genetic suppression of MYC impairing the growth of KRAS-driven cancer cells [87,88]. The MYC oncoprotein in KRAS mutant cells is stabilized via increased MYC transcription and decreased protein degradation mediated by CDK 9 directed phosphorylation of $M Y C$ [89]. Thus, targeting the MYC oncogene could be a potential therapeutic strategy for MYC-dependent cancers such as KRAS-mutant CRC [90]. Voruciclib is a cyclin-dependent kinase (CDK) inhibitor and selectively inhibits cyclin-dependent kinase 4 (CDK4) and 6 (CDK6) [91]. This, in turn, blocks the phosphorylation of the retinoblastoma protein in early G1 phase, preventing the CDK-mediated G1-S phase transition leading to cell cycle arrest. The suppression of replication of DNA, in turn, inhibits tumor cell proliferation. The anti-neoplastic potential of voruciclib arises from its activity to inhibit the Cyclin-dependent Kinases. Wiley et al. presented the pre-clinical study at AACR virtual meeting [92]. The preclinical study showed that when cancer cell lines with KRAS mutations were treated with voruciclib, all cell lines had decrease in viability, and reduced MYC levels were noted. The ability of voruciclib to inhibit tumor growth in vivo was also tested in murine xenograft models injected separately with KRAS mutant CRC, NSCLC primary human cancer cells, wherein significant tumor growth inhibition $(>50 \%)$ was observed at all doses of voruciclib [92]. This promising data from single agent voruciclib supports the hypothesis that the CDK9 inhibitor might synergize with the KRAS $\mathrm{C} 12 \mathrm{C}$ inhibitors sotorasib and adagrasib.

\section{3. $T$ Cell-Mediated Therapy}

Adoptive cell therapy (ACT) uses ex-vivo expanded tumor-reactive T-cells administered to an adequately prepared recipient [93]. This may be the future of RAS directed therapy [94]. Tan et al. in 2016 reported the first CD8+ T-cell response against mutant KRAS G12D in tumor-infiltrating lymphocytes (TILs) obtained from a patient with metastatic colorectal cancer. They reported objective regression of all seven lung metastatic lesions from underlying CRC after the infusion of KRAS G12D directed TILs [95]. RAS mutations represent ideal targets for immune-based treatments. The $\mathrm{T}$ cell mediated therapy represents unique modality that overcomes the many limitations of existing small molecule inhibitors, non-specific immune-based therapies, and passive vaccination trials. T cellmediated therapy could represent the fascinating approach to overcome the prototypical "undruggable" RAS oncogene family. 


\section{Conclusions}

In this review, we presented the promising advances in the development of KRAS directed therapy. KRAS directed therapy seems the most exciting approach, especially with the approval of the KRAS 12C directed agent in NSCLC. In the coming years, we are hopeful to have a similar agent in CRC based on the data from the preclinical and early phase trials. However, it is essential to consider that development of acquired resistance is inevitable. Apart from the KRAS directed therapy, combining it with a downstream pathway inhibitor such as CDK, immunotherapy, or chemotherapy needs further exploration. Ultimately, the tumor stratification is necessary for the success of the KRAS directed therapies. With our evolving knowledge regarding the heterogeneity of the KRAS mutated cancers and multiple subtypes of KRAS mutant forms, the precise selection of the patients for cancerdirected therapy will be necessary to ensure efficacy. Nonetheless, the future of KRAS directed therapy is promising. The data stimulate increasingly more effort to seek a better understanding of overcoming the long-time un-druggable target in oncology.

Author Contributions: Writing—original draft preparation, S.R.; writing—review and editing, R.M. and S.G. (Sanjay Goel); Figure credits-M.G.; Table credits-S.G. (Shimon Garrel); funding acquisition, R.M. and S.G. (Sanjay Goel). All authors have read and agreed to the published version of the manuscript.

Funding: This research was funded from NIH R 21 \# AG058027 02 (NIA) and the start-up fund \#2A4108 (received by Sanjay Goel), as well as from an Alexander Fund 252869-252929 provided by the Yeshiva University Office of the Provost to Radhashree Maitra.

Acknowledgments: We are thankful to the Department of Medical Oncology at the Montefiore Medical Center/ Albert Einstein College of Medicine, New York 10467, USA.

Conflicts of Interest: The authors declare no conflict of interest.

\section{References}

1. Siegel, R.L.; Miller, K.D.; Fuchs, H.E.; Jemal, A. Cancer Statistics, 2021. CA Cancer J. Clin. 2021, 71, 7-33. [CrossRef] [PubMed]

2. Islami, F.; Ward, E.M.; Sung, H.; Cronin, K.A.; Tangka, F.K.L.; Sherman, R.L.; Zhao, J.; Anderson, R.N.; Henley, S.J.; Yabroff, K.R.; et al. Annual Report to the Nation on the Status of Cancer, Part 1: National Cancer Statistics. J. Natl. Cancer Inst. 2021, 113, 1648-1669. [CrossRef]

3. Loeb, L.A.; Springgate, C.F.; Battula, N. Errors in DNA Replication as a Basis of Malignant Changes. Cancer Res. 1974, 34, 2311-2321. [PubMed]

4. Pino, M.S.; Chung, D.C. The Chromosomal Instability Pathway in Colon Cancer. Gastroenterology 2010, 138, 2059-2072. [CrossRef] [PubMed]

5. Vilar, E.; Gruber, S.B. Microsatellite instability in colorectal cancer-The stable evidence. Nat. Rev. Clin. Oncol. 2010, 7, 153-162. [CrossRef] [PubMed]

6. Pancione, M.; Remo, A.; Colantuoni, V. Genetic and Epigenetic Events Generate Multiple Pathways in Colorectal Cancer Progression. Pathol. Res. Int. 2012, 2012, 509348. [CrossRef] [PubMed]

7. La Vecchia, S.; Sebastián, C. Metabolic pathways regulating colorectal cancer initiation and progression. Semin. Cell Dev. Biol 2020, 98, 63-70. [CrossRef] [PubMed]

8. Zhang, Z.; Wang, Y.; Vikis, H.G.; Johnson, L.; Liu, G.; Li, J.; Anderson, M.W.; Sills, R.C.; Hong, H.L.; Devereux, T.R.; et al. Wildtype Kras2 can inhibit lung carcinogenesis in mice. Nat. Genet. 2001, 29, 25-33. [CrossRef] [PubMed]

9. Anderson, M.W.; Reynolds, S.H.; You, M.; Maronpot, R.M. Role of proto-oncogene activation in carcinogenesis. Environ. Health Perspect. 1992, 98, 13-24. [CrossRef] [PubMed]

10. Kranenburg, O. The KRAS oncogene: Past, present, and future. Biochim. Biophys. Acta 2005, 1756, 81-82. [CrossRef] [PubMed]

11. Bos, J.L.; Fearon, E.R.; Hamilton, S.R.; Vries, M.V.d.; van Boom, J.H.; van der Eb, A.J.; Vogelstein, B. Prevalence of ras gene mutations in human colorectal cancers. Nature 1987, 327, 293-297. [CrossRef] [PubMed]

12. Bazan, V.; Agnese, V.; Corsale, S.; Calò, V.; Valerio, M.R.; Latteri, M.A.; Vieni, S.; Grassi, N.; Cicero, G.; Dardanoni, G.; et al. Specific TP53 and/or Ki-ras mutations as independent predictors of clinical outcome in sporadic colorectal adenocarcinomas: Results of a 5-year Gruppo Oncologico dell'Italia Meridionale (GOIM) prospective study. Ann. Oncol. 2005, 16, iv50-iv55. [CrossRef] [PubMed]

13. Überall, I.; Kolář, Z.; Trojanec, R.; Berkovcová, J.; Hajdúch, M. The status and role of ErbB receptors in human cancer. Exp. Mol. Pathol. 2008, 84, 79-89. [CrossRef]

14. Scolnick, E.M.; Rands, E.; Williams, D.; Parks, W.P. Studies on the nucleic acid sequences of Kirsten sarcoma virus: A model for formation of a mammalian RNA-containing sarcoma virus. J. Virol. 1973, 12, 458-463. [CrossRef] [PubMed] 
15. Harvey, J.J. An unidentified virus which causes the rapid production of tumours in mice. Nature 1964, 204, 1104-1105. [CrossRef] [PubMed]

16. Stehelin, D.; Varmus, H.E.; Bishop, J.M.; Vogt, P.K. DNA related to the transforming gene(s) of avian sarcoma viruses is present in normal avian DNA. Nature 1976, 260, 170-173. [CrossRef]

17. Román, M.; Baraibar, I.; López, I.; Nadal, E.; Rolfo, C.; Vicent, S.; Gil-Bazo, I. KRAS oncogene in non-small cell lung cancer: Clinical perspectives on the treatment of an old target. Mol. Cancer 2018, 17, 33. [CrossRef]

18. Hall, A.; Marshall, C.J.; Spurr, N.K.; Weiss, R.A. Identification of transforming gene in two human sarcoma cell lines as a new member of the ras gene family located on chromosome 1. Nature 1983, 303, 396-400. [CrossRef]

19. Wennerberg, K.; Rossman, K.L.; Der, C.J. The Ras superfamily at a glance. J. Cell Sci. 2005, 118, 843-846. [CrossRef] [PubMed]

20. Jančík, S.; Drábek, J.; Radzioch, D.; Hajdúch, M. Clinical Relevance of KRAS in Human Cancers. J. Biomed. Biotechnol. 2010, 2010, 150960. [CrossRef] [PubMed]

21. Hancock, J.F.; Magee, A.I.; Childs, J.E.; Marshall, C.J. All ras proteins are polyisoprenylated but only some are palmitoylated. Cell 1989, 57, 1167-1177. [CrossRef]

22. Seger, R.; Krebs, E.G. The MAPK signaling cascade. FASEB J. 1995, 9, 726-735. [CrossRef]

23. Lambert, J.M.; Lambert, Q.T.; Reuther, G.W.; Malliri, A.; Siderovski, D.P.; Sondek, J.; Collard, J.G.; Der, C.J. Tiam1 mediates Ras activation of Rac by a PI(3)K-independent mechanism. Nat. Cell Biol. 2002, 4, 621-625. [CrossRef]

24. Mukhopadhyay, S.; Vander Heiden, M.G.; McCormick, F. The Metabolic Landscape of RAS-Driven Cancers from biology to therapy. Nat. Cancer 2021, 2, 271-283. [CrossRef]

25. Vaughn, C.P.; Zobell, S.D.; Furtado, L.V.; Baker, C.L.; Samowitz, W.S. Frequency of KRAS, BRAF, and NRAS mutations in colorectal cancer. Genes Chromosomes Cancer 2011, 50, 307-312. [CrossRef]

26. Li, Z.-N.; Zhao, L.; Yu, L.-F.; Wei, M.-J. BRAF and KRAS mutations in metastatic colorectal cancer: Future perspectives for personalized therapy. Gastroenterol. Rep. 2020, 8, 192-205. [CrossRef]

27. Zocche, D.M.; Ramirez, C.; Fontao, F.M.; Costa, L.D.; Redal, M.A. Global impact of KRAS mutation patterns in FOLFOX treated metastatic colorectal cancer. Front. Genet. 2015, 6, 116. [CrossRef] [PubMed]

28. Esteller, M.; González, S.; Risques, R.A.; Marcuello, E.; Mangues, R.; Germà, J.R.; Herman, J.G.; Capellà, G.; Peinado, M.A. K-ras and p16 aberrations confer poor prognosis in human colorectal cancer. J. Clin. Oncol. 2001, 19, 299-304. [CrossRef]

29. Lito, P.; Solomon, M.; Li, L.-S.; Hansen, R.; Rosen, N. Allele-specific inhibitors inactivate mutant KRAS G12C by a trapping mechanism. Science 2016, 351, 604-608. [CrossRef]

30. Canon, J.; Rex, K.; Saiki, A.Y.; Mohr, C.; Cooke, K.; Bagal, D.; Gaida, K.; Holt, T.; Knutson, C.G.; Koppada, N.; et al. The clinical KRAS(G12C) inhibitor AMG 510 drives anti-tumour immunity. Nature 2019, 575, 217-223. [CrossRef] [PubMed]

31. Fakih, M.; Desai, J.; Kuboki, Y.; Strickler, J.H.; Price, T.J.; Durm, G.A.; Falchook, G.S.; Denlinger, C.S.; Krauss, J.C.; Shapiro, G.; et al. CodeBreak 100: Activity of AMG 510, a novel small molecule inhibitor of KRASG12C, in patients with advanced colorectal cancer. J. Clin. Oncol. 2020, 38, 4018. [CrossRef]

32. Hong, D.S.; Fakih, M.G.; Strickler, J.H.; Desai, J.; Durm, G.A.; Shapiro, G.I.; Falchook, G.S.; Price, T.J.; Sacher, A.; Denlinger, C.S.; et al. KRASG12C Inhibition with Sotorasib in Advanced Solid Tumors. N. Engl. J. Med. 2020, 383, 1207-1217. [CrossRef] [PubMed]

33. Hallin, J.; Engstrom, L.D.; Hargis, L.; Calinisan, A.; Aranda, R.; Briere, D.M.; Sudhakar, N.; Bowcut, V.; Baer, B.R.; Ballard, J.A.; et al. The KRAS ${ }^{\mathrm{G} 12 \mathrm{C}}$ Inhibitor MRTX8 ${ }_{49}$ Provides Insight toward Therapeutic Susceptibility of KRAS-Mutant Cancers in Mouse Models and Patients. Cancer Discov. 2020, 10, 54-71. [CrossRef] [PubMed]

34. Sabari, J.K.; Park, H.; Tolcher, A.W.; Ou, S.-H.I.; Garon, E.B.; George, B.; Janne, P.A.; Moody, S.E.; Tan, E.Y.; Sen, S.K.; et al. KRYSTAL-2: A phase I/II trial of adagrasib (MRTX849) in combination with TNO155 in patients with advanced solid tumors with KRAS G12C mutation. J. Clin. Oncol. 2021, 39, TPS146. [CrossRef]

35. Xie, M.; Xu, X.; Fan, Y. KRAS-Mutant Non-Small Cell Lung Cancer: An Emerging Promisingly Treatable Subgroup. Front. Oncol. 2021, 11, 672612. [CrossRef]

36. Therapeutics, M. Mirati Therapeutics Reports Investigational Adagrasib (MRTX849) Preliminary Data Demonstrating Tolerability and Durable Anti-Tumor Activity as Well as Initial MRTX1133 Preclinical Data. 2020. Available online: https: / /ir.mirati.com/press-releases/press-release-details/2020/Mirati-Therapeutics-Reports-Investigational-AdagrasibMRTX849-Preliminary-Data-Demonstrating-Tolerability-and-Durable-Anti-Tumor-Activity-as-well-as-Initial-MRTX1133Preclinical-Data/default.aspx (accessed on 28 October 2021).

37. Addeo, A.; Banna, G.L.; Friedlaender, A. KRAS G12C Mutations in NSCLC: From Target to Resistance. Cancers 2021, $13,2541$. [CrossRef]

38. de Cárcer, G.; Manning, G.; Malumbres, M. From Plk1 to Plk5: Functional evolution of polo-like kinases. Cell Cycle 2011, 10, 2255-2262. [CrossRef] [PubMed]

39. Takai, N.; Hamanaka, R.; Yoshimatsu, J.; Miyakawa, I. Polo-like kinases (Plks) and cancer. Oncogene 2005, 24, 287-291. [CrossRef] [PubMed]

40. Cimino, S.K.; Eng, C. Up-and-Coming Experimental Drug Options for Metastatic Colorectal Cancer. J. Exp. Pharm. 2020, 12, 475-485. [CrossRef] [PubMed]

41. Jayachandran, P.; Elliott, A.; Battaglin, F.; Lou, E.; Zhang, W.; Soni, S.; Arai, H.; Wang, J.; Millstein, J.; Lockhart, A.C.; et al. 473P PLK1 expression and KRAS mutations in colorectal cancer. Ann. Oncol. 2020, 31, S442. [CrossRef] 
42. Valsasina, B.; Beria, I.; Alli, C.; Alzani, R.; Avanzi, N.; Ballinari, D.; Cappella, P.; Caruso, M.; Casolaro, A.; Ciavolella, A.; et al. NMS-P937, an orally available, specific small-molecule polo-like kinase 1 inhibitor with antitumor activity in solid and hematologic malignancies. Mol. Cancer 2012, 11, 1006-1016. [CrossRef] [PubMed]

43. Einstein, D.J.; Choudhury, A.D.; Saylor, P.J.; Patterson, J.C.; Croucher, P.; Ridinger, M.; Erlander, M.G.; Yaffe, M.B.; Bubley, G. A phase II study of onvansertib in combination with abiraterone and prednisone in patients with metastatic castration-resistant prostate cancer (mCRPC). J. Clin. Oncol. 2021, 39, TPS186. [CrossRef]

44. Hiraoka, K.; Inoue, T.; Taylor, R.D.; Watanabe, T.; Koshikawa, N.; Yoda, H.; Shinohara, K.; Takatori, A.; Sugimoto, H.; Maru, Y.; et al. Inhibition of KRAS codon 12 mutants using a novel DNA-alkylating pyrrole-imidazole polyamide conjugate. Nat. Commun. 2015, 6, 6706. [CrossRef]

45. Huang, W.Q.; Lin, Q.; Zhuang, X.; Cai, L.L.; Ruan, R.S.; Lu, Z.X.; Tzeng, C.M. Structure, function, and pathogenesis of SHP2 in developmental disorders and tumorigenesis. Curr. Cancer Drug Targets 2014, 14, 567-588. [CrossRef]

46. Cunnick, J.M.; Meng, S.; Ren, Y.; Desponts, C.; Wang, H.G.; Djeu, J.Y.; Wu, J. Regulation of the mitogen-activated protein kinase signaling pathway by SHP2. J. Biol. Chem. 2002, 277, 9498-9504. [CrossRef]

47. Maroun, C.R.; Naujokas, M.A.; Holgado-Madruga, M.; Wong, A.J.; Park, M. The tyrosine phosphatase SHP-2 is required for sustained activation of extracellular signal-regulated kinase and epithelial morphogenesis downstream from the met receptor tyrosine kinase. Mol. Cell. Biol. 2000, 20, 8513-8525. [CrossRef] [PubMed]

48. Wu, C.J.; O’Rourke, D.M.; Feng, G.S.; Johnson, G.R.; Wang, Q.; Greene, M.I. The tyrosine phosphatase SHP-2 is required for mediating phosphatidylinositol 3-kinase/Akt activation by growth factors. Oncogene 2001, 20, 6018-6025. [CrossRef] [PubMed]

49. You, M.; Yu, D.H.; Feng, G.S. Shp-2 tyrosine phosphatase functions as a negative regulator of the interferon-stimulated Jak/STAT pathway. Mol. Cell Biol 1999, 19, 2416-2424. [CrossRef]

50. Patsoukis, N.; Duke-Cohan, J.S.; Chaudhri, A.; Aksoylar, H.-I.; Wang, Q.; Council, A.; Berg, A.; Freeman, G.J.; Boussiotis, V.A. Interaction of SHP-2 SH2 domains with PD-1 ITSM induces PD-1 dimerization and SHP-2 activation. Commun. Biol. 2020, 3, 128. [CrossRef]

51. Zito, C.I.; Qin, H.; Blenis, J.; Bennett, A.M. SHP-2 Regulates Cell Growth by Controlling the mTOR/S6 Kinase 1 Pathway. J. Biol. Chem. 2007, 282, 6946-6953. [CrossRef] [PubMed]

52. Qu, C.K. The SHP-2 tyrosine phosphatase: Signaling mechanisms and biological functions. Cell Res. 2000, 10, 279-288. [CrossRef] [PubMed]

53. Yuan, X.; Bu, H.; Zhou, J.; Yang, C.-Y.; Zhang, H. Recent Advances of SHP2 Inhibitors in Cancer Therapy: Current Development and Clinical Application. J. Med. Chem. 2020, 63, 11368-11396. [CrossRef]

54. Fedele, C.; Li, S.; Teng, K.W.; Foster, C.; Peng, D.; Ran, H.; Mita, P.; Geer, M.; Hattori, T.; Koide, A.; et al. SHP2 Inhibition Abrogates Adaptive Resistance to KRAS ${ }^{\mathrm{G} 12 \mathrm{C}}$-Inhibition and Remodels the Tumor Microenvironment of KRAS-Mutant Tumors. bioRxiv 2020. bioRxiv:2020.2005.2030.125138. [CrossRef]

55. Wu, F.; Niu, K.; Cui, Y.; Li, C.; Lyu, M.; Ren, Y.; Chen, Y.; Deng, H.; Huang, L.; Zheng, S.; et al. Genome-wide analysis of DNA G-quadruplex motifs across 37 species provides insights into G4 evolution. Commun. Biol. 2021, 4, 98. [CrossRef]

56. Brooks, T.A.; Kendrick, S.; Hurley, L. Making sense of G-quadruplex and i-motif functions in oncogene promoters. FEBS J. 2010, 277, 3459-3469. [CrossRef]

57. Cheng, Y.; Tang, Q.; Li, Y.; Zhang, Y.; Zhao, C.; Yan, J.; You, H. Folding/unfolding kinetics of G-quadruplexes upstream of the P1 promoter of the human BCL-2 oncogene. J. Biol. Chem. 2019, 294, 5890-5895. [CrossRef]

58. Lavrado, J.; Borralho, P.M.; Ohnmacht, S.A.; Castro, R.E.; Rodrigues, C.M.; Moreira, R.; dos Santos, D.J.; Neidle, S.; Paulo, A. Synthesis, G-quadruplex stabilisation, docking studies, and effect on cancer cells of indolo[3,2-b]quinolines with one, two, or three basic side chains. ChemMedChem 2013, 8, 1648-1661. [CrossRef] [PubMed]

59. Porru, M.; Artuso, S.; Salvati, E.; Bianco, A.; Franceschin, M.; Diodoro, M.G.; Passeri, D.; Orlandi, A.; Savorani, F.; D'Incalci, M.; et al. Targeting G-Quadruplex DNA Structures by EMICORON Has a Strong Antitumor Efficacy against Advanced Models of Human Colon Cancer. Mol. Cancer 2015, 14, 2541-2551. [CrossRef]

60. Porru, M.; Zizza, P.; Franceschin, M.; Leonetti, C.; Biroccio, A. EMICORON: A multi-targeting G4 ligand with a promising preclinical profile. Biochim. Biophys. Acta 2017, 1861, 1362-1370. [CrossRef]

61. Chandra, A.; Grecco, H.E.; Pisupati, V.; Perera, D.; Cassidy, L.; Skoulidis, F.; Ismail, S.A.; Hedberg, C.; Hanzal-Bayer, M.; Venkitaraman, A.R.; et al. Erratum: The GDI-like solubilizing factor PDE $\delta$ sustains the spatial organization and signalling of Ras family proteins. Nat. Cell Biol. 2012, 14, 329. [CrossRef]

62. Zhang, H.; Liu, X.-h.; Zhang, K.; Chen, C.-K.; Frederick, J.M.; Prestwich, G.D.; Baehr, W. Photoreceptor cGMP Phosphodiesterase $\delta$ Subunit (PDE $\delta$ ) Functions as a Prenyl-binding Protein. J. Biol. Chem. 2004, 279, 407-413. [CrossRef] [PubMed]

63. Papke, B.; Murarka, S.; Vogel, H.A.; Martín-Gago, P.; Kovacevic, M.; Truxius, D.C.; Fansa, E.K.; Ismail, S.; Zimmermann, G.; Heinelt, K.; et al. Identification of pyrazolopyridazinones as PDE $\delta$ inhibitors. Nat. Commun. 2016, 7, 11360. [CrossRef]

64. Martín-Gago, P.; Fansa, E.K.; Klein, C.H.; Murarka, S.; Janning, P.; Schürmann, M.; Metz, M.; Ismail, S.; Schultz-Fademrecht, C.; Baumann, M.; et al. A PDE68-KRas Inhibitor Chemotype with up to Seven H-Bonds and Picomolar Affinity that Prevents Efficient Inhibitor Release by Arl2. Angew. Chem. Int. Ed. Engl. 2017, 56, 2423-2428. [CrossRef]

65. Klein, C.H.; Truxius, D.C.; Vogel, H.A.; Harizanova, J.; Murarka, S.; Martín-Gago, P.; Bastiaens, P.I.H. PDE $\delta$ inhibition impedes the proliferation and survival of human colorectal cancer cell lines harboring oncogenic KRas. Int. J. Cancer 2019, 144, 767-776. [CrossRef] [PubMed] 
66. Maitra, R.; Ghalib, M.H.; Goel, S. Reovirus: A targeted therapeutic-progress and potential. Mol. Cancer Res. 2012, 10, $1514-1525$. [CrossRef]

67. Schmitz, K.J.; Ademi, C.; Bertram, S.; Schmid, K.W.; Baba, H.A. Prognostic relevance of autophagy-related markers LC3, p62/sequestosome 1, Beclin-1 and ULK1 in colorectal cancer patients with respect to KRAS mutational status. World J. Surg. Oncol 2016, 14, 189. [CrossRef]

68. Jiffry, J.; Thavornwatanayong, T.; Rao, D.; Fogel, E.J.; Saytoo, D.; Nahata, R.; Guzik, H.; Chaudhary, I.; Augustine, T.; Goel, S.; et al. Oncolytic Reovirus (pelareorep) Induces Autophagy in KRAS-mutated Colorectal Cancer. Clin. Cancer Res. 2021, 27, 865-876. [CrossRef]

69. Goel, S.; Ocean, A.J.; Parakrama, R.Y.; Ghalib, M.H.; Chaudhary, I.; Shah, U.; Viswanathan, S.; Kharkwal, H.; Coffey, M.; Maitra, R. Elucidation of Pelareorep Pharmacodynamics in A Phase I Trial in Patients with KRAS-Mutated Colorectal Cancer. Mol. Cancer Ther. 2020, 19, 1148-1156. [CrossRef] [PubMed]

70. Allen, L.F.; Sebolt-Leopold, J.; Meyer, M.B. CI-1040 (PD184352), a targeted signal transduction inhibitor of MEK (MAPKK). Semin. Oncol. 2003, 30, 105-116. [CrossRef]

71. Carracedo, A.; Pandolfi, P.P. The PTEN-PI3K pathway: Of feedbacks and cross-talks. Oncogene 2008, 27, 5527-5541. [CrossRef] [PubMed]

72. Ross, S.J.; Revenko, A.S.; Hanson, L.L.; Ellston, R.; Staniszewska, A.; Whalley, N.; Pandey, S.K.; Revill, M.; Rooney, C.; Buckett, L.K.; et al. Targeting KRAS-dependent tumors with AZD4785, a high-affinity therapeutic antisense oligonucleotide inhibitor of KRAS. Sci. Transl. Med. 2017, 9. [CrossRef]

73. Jones, D. The long march of antisense. Nat. Rev. Drug Discov. 2011, 10, 401-402. [CrossRef]

74. Yeh, T.C.; Marsh, V.; Bernat, B.A.; Ballard, J.; Colwell, H.; Evans, R.J.; Parry, J.; Smith, D.; Brandhuber, B.J.; Gross, S.; et al. Biological characterization of ARRY-142886 (AZD6244), a potent, highly selective mitogen-activated protein kinase kinase $1 / 2$ inhibitor. Clin. Cancer Res. 2007, 13, 1576-1583. [CrossRef] [PubMed]

75. Davies, B.R.; Logie, A.; McKay, J.S.; Martin, P.; Steele, S.; Jenkins, R.; Cockerill, M.; Cartlidge, S.; Smith, P.D. AZD6244 (ARRY-142886), a potent inhibitor of mitogen-activated protein kinase/extracellular signal-regulated kinase kinase 1/2 kinases: Mechanism of action in vivo, pharmacokinetic/pharmacodynamic relationship, and potential for combination in preclinical models. Mol. Cancer 2007, 6, 2209-2219. [CrossRef]

76. Bennouna, J.; Lang, I.; Valladares-Ayerbes, M.; Boer, K.; Adenis, A.; Escudero, P.; Kim, T.-Y.; Pover, G.M.; Morris, C.D.; Douillard, J.-Y. A Phase II, open-label, randomised study to assess the efficacy and safety of the MEK1/2 inhibitor AZD6244 (ARRY-142886) versus capecitabine monotherapy in patients with colorectal cancer who have failed one or two prior chemotherapeutic regimens. Investig. New Drugs 2011, 29, 1021-1028. [CrossRef]

77. Ciombor, K.K.; Bekaii-Saab, T. Selumetinib for the treatment of cancer. Expert Opin. Investig. Drugs 2015, 24, 111-123. [CrossRef] [PubMed]

78. Shimizu, T.; Tolcher, A.W.; Papadopoulos, K.P.; Beeram, M.; Rasco, D.W.; Smith, L.S.; Gunn, S.; Smetzer, L.; Mays, T.A.; Kaiser, B.; et al. The clinical effect of the dual-targeting strategy involving PI3K/AKT/mTOR and RAS/MEK/ERK pathways in patients with advanced cancer. Clin. Cancer Res. 2012, 18, 2316-2325. [CrossRef] [PubMed]

79. Pitts, T.M.; Newton, T.P.; Bradshaw-Pierce, E.L.; Addison, R.; Arcaroli, J.J.; Klauck, P.J.; Bagby, S.M.; Hyatt, S.L.; Purkey, A.; Tentler, J.J.; et al. Dual pharmacological targeting of the MAP kinase and PI3K/mTOR pathway in preclinical models of colorectal cancer. PLoS ONE 2014, 9, e113037. [CrossRef]

80. Martinelli, E.; Troiani, T.; D’Aiuto, E.; Morgillo, F.; Vitagliano, D.; Capasso, A.; Costantino, S.; Ciuffreda, L.P.; Merolla, F.; Vecchione, L.; et al. Antitumor activity of pimasertib, a selective MEK 1/2 inhibitor, in combination with PI3K/mTOR inhibitors or with multi-targeted kinase inhibitors in pimasertib-resistant human lung and colorectal cancer cells. Int. J. Cancer 2013, 133, 2089-2101. [CrossRef]

81. E, J.; Xing, J.; Gong, H.; He, J.; Zhang, W. Combine MEK inhibition with PI3K/mTOR inhibition exert inhibitory tumor growth effect on KRAS and PIK3CA mutation CRC xenografts due to reduced expression of VEGF and matrix metallopeptidase-9. Tumor Biol. 2015, 36, 1091-1097. [CrossRef]

82. Ha, M.; Kim, V.N. Regulation of microRNA biogenesis. Nat. Rev. Mol. Cell Biol 2014, 15, 509-524. [CrossRef] [PubMed]

83. Peng, Y.; Croce, C.M. The role of MicroRNAs in human cancer. Signal. Transduct. Target. Ther. 2016, 1, 15004. [CrossRef] [PubMed]

84. Hong, Y.; Liang, H.; Uzair Ur, R.; Wang, Y.; Zhang, W.; Zhou, Y.; Chen, S.; Yu, M.; Cui, S.; Liu, M.; et al. miR-96 promotes cell proliferation, migration and invasion by targeting PTPN9 in breast cancer. Sci. Rep. 2016, 6, 37421. [CrossRef] [PubMed]

85. Chen, X.; Guo, X.; Zhang, H.; Xiang, Y.; Chen, J.; Yin, Y.; Cai, X.; Wang, K.; Wang, G.; Ba, Y.; et al. Role of miR-143 targeting KRAS in colorectal tumorigenesis. Oncogene 2009, 28, 1385-1392. [CrossRef] [PubMed]

86. Zhou, Y.; Dang, J.; Chang, K.Y.; Yau, E.; Aza-Blanc, P.; Moscat, J.; Rana, T.M. miR-1298 Inhibits Mutant KRAS-Driven Tumor Growth by Repressing FAK and LAMB3. Cancer Res. 2016, 76, 5777-5787. [CrossRef]

87. Soucek, L.; Whitfield, J.R.; Sodir, N.M.; Massó-Vallés, D.; Serrano, E.; Karnezis, A.N.; Swigart, L.B.; Evan, G.I. Inhibition of Myc family proteins eradicates KRas-driven lung cancer in mice. Genes Dev. 2013, 27, 504-513. [CrossRef] [PubMed]

88. Soucek, L.; Whitfield, J.; Martins, C.P.; Finch, A.J.; Murphy, D.J.; Sodir, N.M.; Karnezis, A.N.; Swigart, L.B.; Nasi, S.; Evan, G.I. Modelling Myc inhibition as a cancer therapy. Nature 2008, 455, 679-683. [CrossRef] 
89. Blake, D.R.; Vaseva, A.V.; Hodge, R.G.; Kline, M.P.; Gilbert, T.S.K.; Tyagi, V.; Huang, D.; Whiten, G.C.; Larson, J.E.; Wang, X.; et al. Application of a MYC degradation screen identifies sensitivity to CDK9 inhibitors in KRAS-mutant pancreatic cancer. Sci. Signal. 2019, 12. [CrossRef] [PubMed]

90. Ischenko, I.; Zhi, J.; Hayman, M.J.; Petrenko, O. KRAS-dependent suppression of MYC enhances the sensitivity of cancer cells to cytotoxic agents. Oncotarget 2017, 8, 17995-18009. [CrossRef]

91. Gupta, P.; Zhang, Y.K.; Zhang, X.Y.; Wang, Y.J.; Lu, K.W.; Hall, T.; Peng, R.; Yang, D.H.; Xie, N.; Chen, Z.S. Voruciclib, a Potent CDK4/6 Inhibitor, Antagonizes ABCB1 and ABCG2-Mediated Multi-Drug Resistance in Cancer Cells. Cell. Physiol. Biochem. 2018, 45, 1515-1528. [CrossRef] [PubMed]

92. Wiley, S.E.; Su, Y.; Ge, Y. Abstract 1962: Voruciclib, a CDK9 inhibitor, downregulates MYC and inhibits proliferation of KRAS mutant cancers in preclinical models. Cancer Res. 2021, 81, 1962. [CrossRef]

93. Lu, Y.-C.; Yao, X.; Crystal, J.S.; Li, Y.F.; El-Gamil, M.; Gross, C.; Davis, L.; Dudley, M.E.; Yang, J.C.; Samuels, Y.; et al. Efficient Identification of Mutated Cancer Antigens Recognized by T Cells Associated with Durable Tumor Regressions. Clin. Cancer Res. 2014, 20, 3401-3410. [CrossRef] [PubMed]

94. T-Cell Transfer Therapy Targeting Mutant KRAS. N. Engl. J. Med. 2017, 376, e11. [CrossRef] [PubMed]

95. Tran, E.; Robbins, P.F.; Lu, Y.-C.; Prickett, T.D.; Gartner, J.J.; Jia, L.; Pasetto, A.; Zheng, Z.; Ray, S.; Groh, E.M.; et al. T-Cell Transfer Therapy Targeting Mutant KRAS in Cancer. N. Engl. J. Med. 2016, 375, 2255-2262. [CrossRef] [PubMed] 